\title{
Response Inhibition and Emotional Regulation in the Patients with Attention-Deficit/Hyperactivity Disorder and Comorbidity of Disruptive, Impulse-Control, and Conduct Disorders
}

\author{
Xixi Jiang ${ }^{1,2}$, Yuncheng Zhu ${ }^{2}$, and Yiru Fang ${ }^{2,3,4 凶}$ \\ ${ }^{1}$ Department of Psychosomatic Medicine, Shanghai Changning Mental Health Center, Affiliated Greenland Hospital of Bio-X Institute, \\ Shanghai Jiao Tong University, Shanghai, China \\ ${ }^{2}$ Clinical Research Center, Division of Mood Disorders, Shanghai Mental Health Center, Shanghai Jiao Tong University School of Medicine, \\ Shanghai, China \\ ${ }^{3}$ CAS Center for Excellence in Brain Science and Intelligence Technology, Shanghai, China \\ ${ }^{4}$ Shanghai Key Laboratory of Psychotic Disorders, Shanghai, China
}

In the previous research published by Stahl SM, five cortico-striato-thalamo-cortical (CSTC) circuits correlated with attention-deficit/hyperactivity disorder (ADHD) were observed. ${ }^{1}$ Dorsolateral CSTC circuit for being recognized as the sustained attention circuit, ventrolateral CSTC circuit as the emotion circuit, anterior cingulate CSTC circuit as the selective attention circuit, motor CSTC circuit as the hyperactivity circuit and orbitofrontal CSTC circuit as the compulsivity \& impulsivity circuit.

It is worth to note that, except for the emotional symptom generating from the ventrolateral CSTC circuit, the effects on $\mathrm{ADHD}$ are easy to associate with the diagnostic components in the Diagnostic and Statistical Manual of Mental Disorders, 5th Edition (DSM-5) from the other four circuits. Interestingly, we found that the pathogenicity of the emotion circuit is also closely related with $\mathrm{ADHD}, 2,3$ because severe affective problems in disruptive, impulse-control, and conduct disorders (DICCD) results from this circuit managing emotional response as well. ${ }^{4}$

Received: May 13, 2019 Revised: July 26, 2019

Accepted: September 30, 2019

$\square$ Correspondence: Yiru Fang, MD, PhD

Division of Mood Disorders, Shanghai Mental Health Center, Shanghai Jiao Tong University School of Medicine, 600 South Wan Ping Road, Shanghai 200030, China

Tel: +86-021-64387250, Fax: +86-021-64387986

E-mail: yirufang@aliyun.com

Yuncheng $\mathrm{Zhu}, \mathrm{MD}, \mathrm{PhD}$

Division of Mood Disorders, Shanghai Mental Health Center, Shanghai Jiao Tong University School of Medicine, 600 South Wan Ping Road, Shanghai 200030, China

Tel: +86-021-64387250, Fax: +86-021-64387986

E-mail: hellfiregenius@163.com

(a) This is an Open Access article distributed under the terms of the Creative Commons Attribution Non-Commercial License (https://creativecommons.org/licenses/by$\mathrm{nc} / 4.0$ ) which permits unrestricted non-commercial use, distribution, and reproduction in any medium, provided the original work is properly cited.
Executive function $(\mathrm{EF})$ can be divided into response inhibition and metacognition. Response inhibition needs the ability to inhibit cognitive and emotional activities. In turn, deficits in response inhibition contribute to four aspects of EF in the metacognition, which includes planning, problem-solving, emotional self-regulation, and nonverbal/verbal working memory. Therefore, we extracted the response inhibition and emotional self-regulation for study. Besides, the EF model of ADHD has been subdivided into two theoretical frameworks, called "cool" and "hot" EF. In brief, the "cool" EF applies to the pure cognitive processing, which solves the problems of the abstraction and de-contextualization related to the sustained attention and selective attention circuit. The "hot" EF takes in charge of the cognitive processing over emotional response and decision-making processing, which is relevant to the emotion and compulsivity \& impulsivity circuit. ${ }^{5}$ For different research purpose, the Stroop test is designed as many classic paradigm of "cool" EF. ${ }^{2}$ Moreover, the emotional Stroop test was used as an extraordinary modified version of the Stroop test. Based on the original advantages of the Stroop effect, affective words from the examined Chinese thesaurus were packed into the emotional Stroop test as incongruent, congruent or neural words with different colors, and then, the emotional responses can be pinpointed simultaneously. If confirmed, cognitive conflict and emotional response may play two different roles in superposition for emotional traits of subjects. ${ }^{5}$

In the recent years, studies have focused on the association between the callous-unemotional (CU) traits and oppositional defiant disorder (ODD) or conduct disorder (CD). The CU traits consist of these personality traits: lack of empathy, remorse or guilt, callous conduct, superficialness and affection- 
lessness. Recently, the CU traits are considered to be the early stage characteristics of DICCD. Therefore, it can provide us with a theoretical framework for bridging gaps between neuropsychology and clinical comorbidity.

Our study showed statistical differences on both the errors and reaction time in Golden Stroop test and the MRT in emotional Stroop test. The yielded difference of response inhibition deficit, as well as the emotional responding, are the core symptoms of ADHD.

The outcome of Golden Stroop test indicated that the ODD/ CD group had no statistical significance from the HC group when we did not put the emotional response into consideration. However, after the incongruent element of emotional response blueprinted into our test tool, the response inhibition of the ODD/CD group was significantly different from that of the HC group, especially in the negative-inconguency block, which was the most seriously affected element and of no difference from that in the ADHD-related group. The different outcome separated by the Golden Stroop test and the emotional Stroop test suggests that the bias of emotional stimuli may be responsible for the $\mathrm{CU}$ traits of ODD/CD that take oversuppressed effects on the activity of response inhibition, particularly in those ODD/CD subjects processing with negative emotions on the course of these Stroop tests. ${ }^{5}$ The recorded aberrant processing of negative emotional response is likely to be related with the $\mathrm{CU}$ traits in the course of response inhibition, particularly in ADHD. The above mentioned results suggested that the emotional Stroop interference effect in ODD/ CD was less influenced than in ADHD.

Another outcome is noteworthy that there is no difference of Golden Stroop IG between the comorbidity group and the ODD/CD group in the Golden Stroop test. We found that the patients with comorbidity were less influenced in response inhibition than in the ADHD group. The probable reason may be that the CU traits are more responsible for the clinical characteristics of comorbidity.

The CU traits have the familiar span of time and stability with other personality traits. Many researches on the specificity of CU traits were displayed at neuropsychological level. The CU traits especially attenuate the awareness of sorrowful images compared to that of other types of images (neutral images, positive images, dangerous situation pictures) with the same results showed in self-reports. The maturation process of $\mathrm{CU}$ traits is different from that of unaffected peers, where cognitive empathy of DICCD turns out to be improved with age but emotional empathy is still insensitive and flawed. The CU traits on the cognition of DICCD also have deficits in responding to rewards and punishments that compared to unaffected children (5), their behavior are usually reward-oriented and insensitive to penalty.
In recent times, studies on this special topic have recognized that the CU traits play a critical role in the classification of DICCD, including ODD and CD. Therefore, DSM-5 not only followed DSM-IV by classification and description of CD symptoms to describe DICCD, but also the CU traits were enumerated as a characteristic factor to make diagnosis more specific. With-or-without CU traits have significant differences in many conditions of pathology, cognitive behavior, disease development and prognosis. Aforesaid traits include pursuit own interests, insensitivity to peer's fear and sadness, elevated externalizing behaviors, lack of empathy, and fearlessness. These CU traits provide patients with no scruples about indifferent to punishment, peer's communication problems, oppositionality-defiance, and more prone to criminal behaviors, which increase the hardship of multicomponent medical interventions. Therefore, it may be regarded as an essential factor for recognizing aggressive and antisocial behavior to distinguish the inventory of CU traits with identified CU dimensions (Unemotional, Callous, Uncaring) in DICCD.

Although the results of this study are interesting, the sample size is small. Therefore, the statistical power is limited. It should be very careful for us to draw a conclusion from this pretest. We are looking forward to expanding the sample size for improving the originality and significant of the series of study.

Our study was based on approval by the Institutional Ethical Committee for clinical research of Shanghai Changning Mental Health Center, Shanghai, China (No. M201801) according to the Declaration of Helsinki.

\section{Acknowledgments}

This work was supported by the National Key R\&D Program of China (2016YFC1307100); the National Natural Science Foundation (81771465, 91232719); the Sanming Project of Medicine in Shenzheng (SZSM201612006); and the Research Project of Shanghai Changning District Science and Technology Commission (CNKW2018Y23).

\section{Conflicts of Interest}

The authors have no potential conflicts of interest to disclose.

\section{Author Contributions}

Xixi Jiang designed the study, collected and analyzed data and wrote the first draft of the manuscript. Yuncheng Zhu contributed to data analysis, translation and manuscript revision. Yiru Fang reviewed the manuscript.

\section{ORCID iDs}

Yiru Fang

Yuncheng Zhu

Xixi Jiang https://orcid.org/0000-0002-8748-9085
https://orcid.org/0000-0003-4686-8887
https://orcid.org/0000-0001-9739-7152

\section{REFERENCES}

1. Stahl SM. Stahl's Essential Psychopharmacology: Neuroscientific Basis and Practical Applications: Cambridge: Cambridge University Press; 2013.

2. Zhu Y, Yang D, Ji W, Huang T, Xue L, Jiang X, et al. The relationship between neurocircuitry dysfunctions and attention deficit hyperactivi- 
ty disorder: a review. Biomed Res Int 2016;2016:3821579.

3. Jiang X, Liu L, Ji H, Zhu Y. Association of affected neurocircuitry with deficit of response inhibition and delayed gratification in attention deficit hyperactivity disorder: a narrative review. Front Hum Neurosci 2018;12:506.

4. Zhu Y, Jiang X, Ji W. The mechanism of cortico-striato-thalamo-cortical neurocircuitry in response inhibition and emotional responding in attention deficit hyperactivity disorder with comorbid disruptive behavior disorder. Neurosci Bull 2018;34:566-572.

5. Jiang X, Liu L, Ji H, Gao J, Zhang M, Zhu Y, et al. Response inhibition and emotional responding in attention-deficit/hyperactivity disorder with comorbid disruptive, impulse-control, and conduct disorders. Nan Fang Yi Ke Da Xue Xue Bao 2019;39:30-34. 